\title{
RF deflecting cavity for fast radioactive ion beams
}

\author{
S. V. Kutsaev ${ }^{1 *}$ (D) A. S. Plastun ${ }^{2}$, R. Agustsson ${ }^{1}$, D. Bazin ${ }^{3,4}$, N. Bultman², P. N. Ostroumov ${ }^{2}$, A. Y. Smirnov ${ }^{1}$, K. Taletski $^{1}$, \\ O. Tarasov ${ }^{3}$ and R. G. T. Zegers $3,4,5$
}

* Correspondence: kutsaev@ radiabeam.com

${ }^{1}$ RadiaBeam Technologies LLC, 1717 Stewart St, Santa Monica, CA 90404, USA

Full list of author information is available at the end of the article

\begin{abstract}
The Facility for Rare Isotope Beams (FRIB) will be a new scientific user facility that produces rare-isotope beams for experiments from the fragmentation of heavy ions at energies of 100-200 MeV/u. During the projectile fragmentation, the rare isotope of interest is produced along with many contaminants that need to be removed before the beam reaches detectors. At FRIB, this is accomplished with a magnetic projectile fragment separator. However, to achieve higher beam purity, in particular for proton-rich rare isotopes, additional purification is necessary. RadiaBeam in collaboration with Michigan State University (MSU) has designed a $20.125 \mathrm{MHz}$ radiofrequency (RF) fragment separator capable of producing a $4 \mathrm{MV}$ kick with $18 \mathrm{~cm}$ aperture in order to remove contaminant isotopes based on their time of flight. In this paper, we will discuss the RF and engineering design considerations of this separator cavity.
\end{abstract}

Keywords: Radioactive beams, Heavy ions, Rare isotopes, Fragment separator, RF deflector, FRIB;

\section{Springer Open}

\section{Introduction}

Radioactive beams have a major impact on studies of nuclear structure and nuclear physics far from stability [1]. The ability to measure and model the properties of unstable nuclei is also of high interest because they play a key role in stellar nuclear reactions, including those that form all heavy elements and the formation and structure of neutron stars [2]. Experiments with rare-isotope beams are performed at a wide variety of institutions around the world. Soon, the most powerful such facility in the world, RIBF [3] in Japan, will be joined by two other next-generation facilities, FAIR [4] in Germany and FRIB [5] in the United States.

The Facility for Rare Isotope Beams (FRIB) will be a cutting-edge research facility to enable breakthrough discoveries in the physics of nuclei, nuclear astrophysics, fundamental interactions, and applied sciences [6-8]. The heart of FRIB is a high-power superconducting linear accelerator that will accelerate primary ion beams up to $200 \mathrm{MeV} / \mathrm{u}$ (for uranium) to strike a target, creating rare isotopes (https://www.frib.msu.edu) [9]. The rare

(c) The Author(s); licensee Springer on behalf of EPJ. 2020, corrected publication 2021. Open Access This article is licensed under a Creative Commons Attribution 4.0 International License, which permits use, sharing, adaptation, distribution and reproduction in any medium or format, as long as you give appropriate credit to the original author(s) and the source, provide a link to the Creative Commons licence, and indicate if changes were made. The images or other third party material in this article are included in the article's Creative Commons licence, unless indicated otherwise in a credit line to the material. If material is not included in the article's Creative Commons licence and your intended use is not permitted by statutory regulation or exceeds the permitted use, you will need to obtain permission directly from the copyright holder. To view a copy of this licence, visit http://creativecommons.org/ licenses/by/4.0\%. 
isotopes are then transported to the relevant vault for performing a wide variety of experiments, such as reaction studies and decay spectroscopy.

During the projectile fragmentation isotope production, along with the desired rare isotope, many other isotope species are produced. To maximize the FRIB scientific program, the secondary fragments must be filtered out to ensure the delivery of rare isotopes with high rates and high purities [10]. The key part of FRIB will be a nextgeneration three-stage magnetic projectile fragment separator, specifically designed to handle the very intense primary and secondary beams [11].

Separation by magnetic rigidity is very effective on the neutron-rich side of the chart of the isotopes, but less effective for proton-rich nuclei [12], due to rigidity overlap. Therefore, the low momentum tails of the contaminants overlap with the optimum rigidity of the fragment of interest. These low momentum tails come from dissipative effects in the fragmentation reaction, and tend to get smaller with increasing energy. They are non-existent on the high momentum side, which is why this problem doesn't occur on the neutron-rich side. For further beam purification on the proton-rich side, it is necessary to have an additional method for purifying the beam. This can be realized through the time of flight separation [13] using an RF fragment separator (RFFS), sometimes referred to as an RF deflector [14]. The operational concept of such a device is shown in Fig. 1. In the RF separation technique, a time difference between beam components develops due to the velocity difference, which results in a different deflection by a time-dependent electromagnetic transverse RF field. This happens because the fragments arrive at different phases of the deflecting RF field in the cavity. Out-ofphase fragments are deflected from the beam axis and then removed by the slit.

In order to account for possible radio-activation of the separator components, the beam intensity will be monitored. This will be done prior and after the cavity. However, beam intensities near the cavity will be limited because the beams are initially purified in the FRIB Fragment Separator. For example, for the High Rigidity Spectrometer (HRS) the beam intensities will not exceed $\sim 10^{7} \mathrm{pps}$ at the cavity and activation is very limited and mostly short-lived. Hence, remote handling near the cavity is not required.

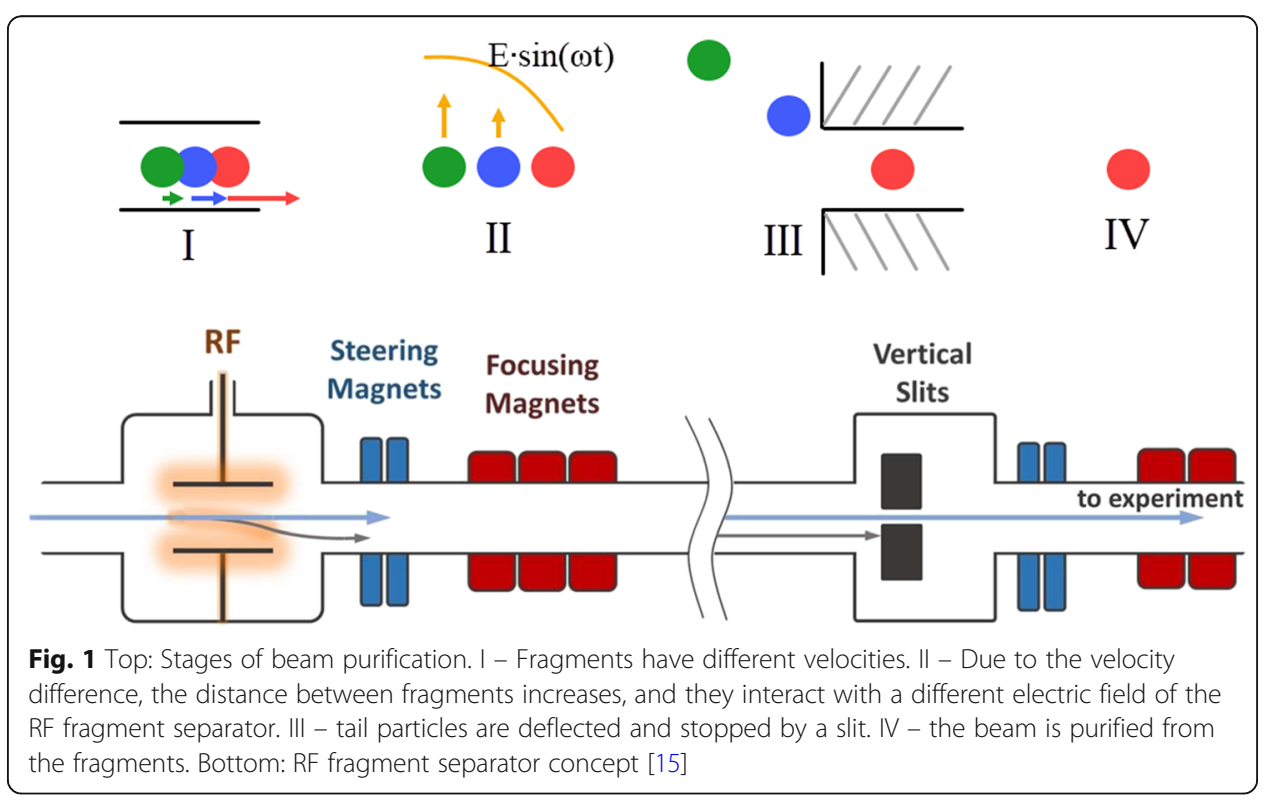


RadiaBeam, in collaboration with FRIB and National Superconducting Cyclotron Laboratory (NSCL) scientists at Michigan State University, has designed a RF fragment separator capable of producing a $4 \mathrm{MV}$ transverse kick, defined as $V_{T}=\int_{0}^{L} E_{T}(z) \cdot \cos (\omega t) \cdot d z$. Here, $E_{T}$ is the transverse electric field, $\omega$ is the RF angular frequency and $z$ is the beam direction. Also, it is necessary to avoid neighboring bunches mixing due to widening in the time difference before entering the separator cavity. This can be done by increasing the inter-bunch distance. For this mode FRIB will provide $20.125 \mathrm{MHz}$ beams (see Fig. 2) [16]. The beams will be prebunched at $40.25 \mathrm{MHz}$ using the existing multi-harmonic buncher. An RF chopper located upstream of the SRF part of the linac will remove every other bunch creating the $20.125 \mathrm{MHz}$ bunch structure. The chopper is not in the baseline lattice of FRIB and will be an additional component. An example of how the separation works is shown in Fig. 3. In both panels, the deflection produced by the RFFS is plotted against the time of flight to the RF fragment separator. The desired isotope is ${ }^{100} \mathrm{Sn}$ (one of the most desirable proton-rich isotopes for experiments at FRIB), which is produced at a very minor fraction compared to the contaminants. On the right-hand side, two out of four beam buckets are filled and even when using deflection position slits (y-axis) to select ${ }^{100} \mathrm{Sn}$, contaminations cannot be removed effectively, because the RF bunches overlap. When three buckets are removed (left-hand side of Fig. 3), it is possible to select ${ }^{100} \mathrm{Sn}$ isotopes effectively, with only one or two weaker contaminant isotope species remaining.

Based on LISE++ beam dynamics simulations [17] shown in the figure, it is possible to increase the separation efficiency by a factor of $300[18,19]$. This is sufficient for reducing the number of contaminants such that particle identification detectors can be used to identify the beam particle on an event-by-event case and thus isolate events induced by ${ }^{100} \mathrm{Sn}$ only. There is no reduction of the ${ }^{100} \mathrm{Sn}$ production rate from the momentum acceptance of the RFFS, but reduction is possible from the transverse momentum spread if the transverse emittance is larger than that for which the RF separator gap was designed.

The simulations, shown in Fig. 3 used the momentum acceptance of $\pm 1.5 \%$ or $3 \%$ spread in fractional momentum deviation. The momentum compression was used in the calculations, so that the actual momentum acceptance is larger than this number. Increasing the momentum acceptance will increase the overlap in time-of-flight, therefore reduce the filtering capability of the RF separator. A compromise has to be reached between increasing the rate by opening the momentum acceptance, and losing purity, which ultimately depends on the experiment's goals. More details about beam

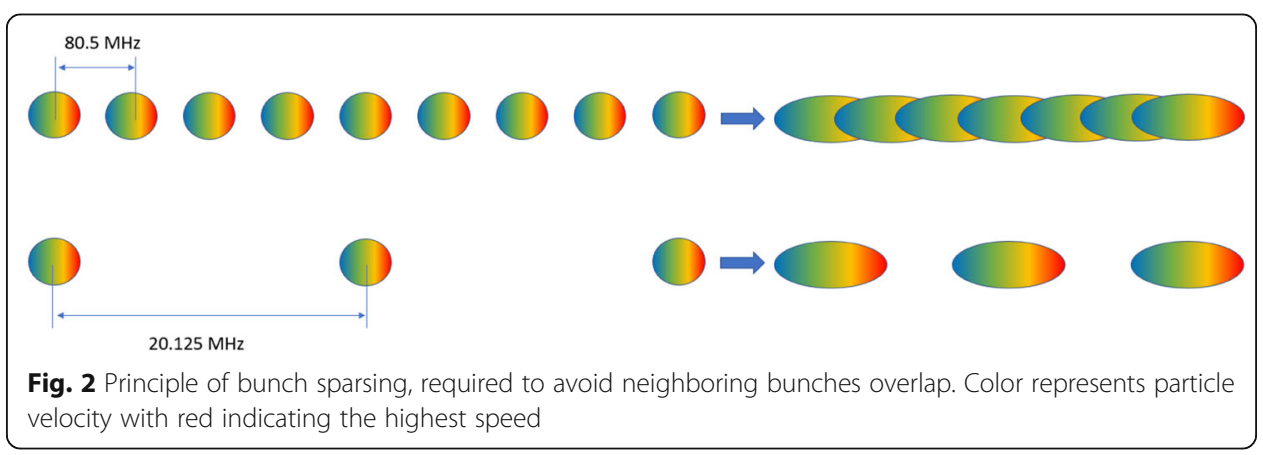



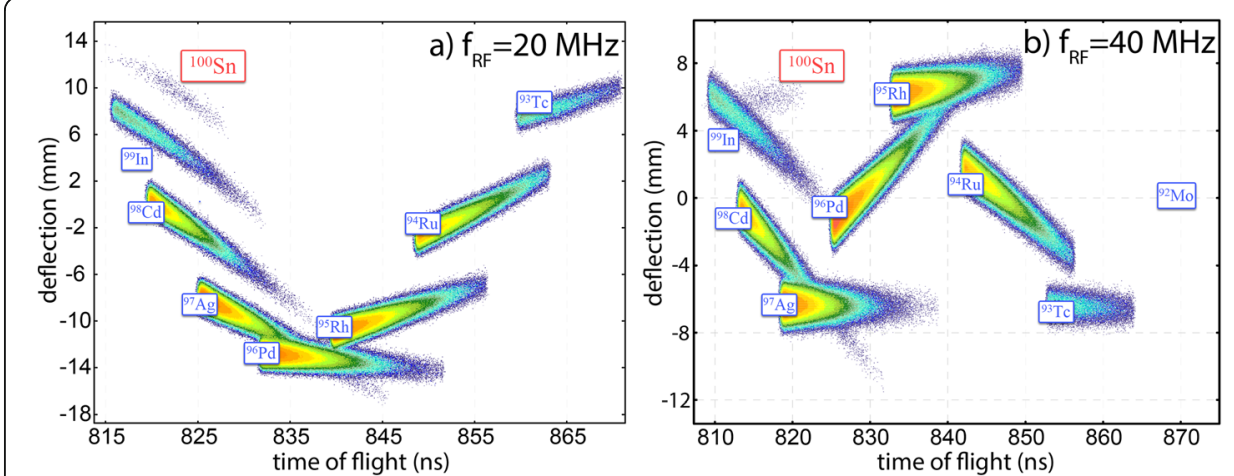

Fig. 3 The purification of a ${ }^{100} \mathrm{Sn}$ beam simulated in LISE++ for two operating frequencies of the RFFS: a $20 \mathrm{MHz}$ (left) and b $40 \mathrm{MHz}$ (right). The figures show the distributions just after the cavity and still prior to slits used for cutting the contaminants

dynamics in the RF separator can be found in the Conceptual Design Report of the FRIB High Rigidity Spectrometer [20]. In this paper we will focus on the electromagnetic and engineering design of RFFS.

In order to achieve the required kick, it is necessary to produce a $300 \mathrm{kV}$ voltage between electrodes, separated by an $18 \mathrm{~cm}$ aperture, in a $2.6 \mathrm{~m}$-long separator cavity. The chosen aperture dimension does not restrict the acceptance even if the RFFS is not used. The cavity dimensions were chosen due to the beamline space restrictions at FRIB. Generating a compact design for such a low-frequency cavity to fit the existing beamline is a challenging task. We explored different candidate RF structures of the separator, including a split-coaxial resonator (SCR), single and double quarter-wave resonator (QWR and DQWR, respectively) and $\mathrm{H}$-resonators ( $\mathrm{IH}$ - and $\mathrm{CH}-$-). After the comparison, we selected the DQWR as the optimal compromise for fabrication and operations. The reasons for this choice will be covered in the forecoming Sections of this paper. The conceptual design of the designed separator is shown in Fig. 4.

We performed detailed electromagnetic design optimization to reduce the RF power losses and ensure stable operational parameters (such as peak E- field). We used beam dynamics simulations to verify that the beam is deflected by $13 \mathrm{~mm}$ or $8.6 \mathrm{mrad}$ after going through the cavity, per FRIB requirements [20]. The conceptual engineering design, which includes the vacuum chamber, RF cavities, electrodes, couplers, tuners, and cooling systems, was developed. It included structural, thermal, and computational fluid dynamics (CFD) analysis to ensure stable operation. The following sections will describe the separator design in detail.

\section{Method/experimental}

\section{Deflecting structure choice}

As a part of the electromagnetic design of the separator cavity, we performed a detailed survey and preliminary design of different deflecting structures in order to identify the optimal one that can provide the required voltage kick within the dimensional constraints. This choice was a compromise between required RF power, compactness, and fabrication feasibility. In particular, the FRIB beamline sets the following constraints: the length of the separator from flange to flange should not exceed $2.6 \mathrm{~m}$, the distance 


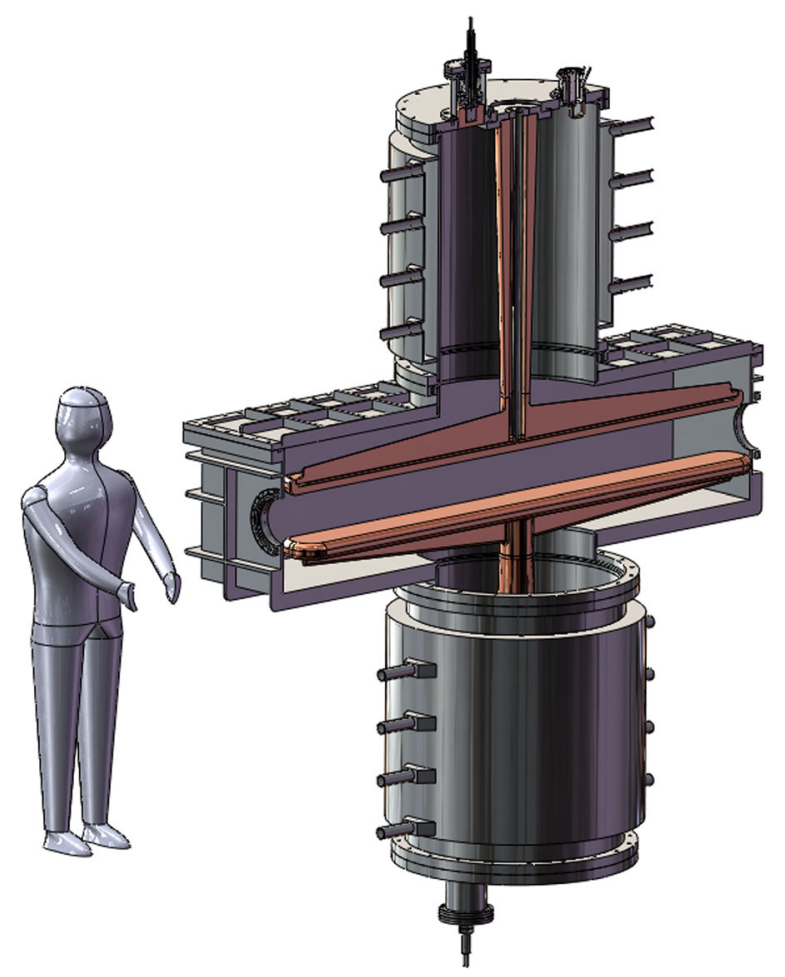

Fig. 4 Conceptual mechanical design of the $20.125 \mathrm{MHz}$ fragment separator cavity. Human is placed for scale

from the bottom to beamline should not exceed $1.5 \mathrm{~m}$, the cavity should operate at $20.125 \mathrm{MHz}$, the aperture size must be $18 \mathrm{~cm}$, and the voltage between electrodes $300 \mathrm{kV}$. Three main classes of resonators were considered: H-resonator, split coaxial resonator and TEM-class resonator.

In H- (or TE-) resonators, shown in Fig. 5, the electrodes load RF cavities and create the voltage across the gap. Depending on the shape of the electrodes, this class of resonator can be subdivided into two subclasses: $\mathrm{CH}$ - (electrode has a shape of the letter $\mathrm{C}$, as shown in Fig. 5b) and IH - (electrode has a shape of the letter I, as shown in Fig. 5a). For such a low frequency, H-resonators have proven to be one of the most efficient cavity types [21].

We started with the study of the IH-resonator due to its mechanical simplicity: it has a stable central stem, and plenty of space for tuners and couplers. This cavity, however, has no built-in adjustability to ensure electrodes parallelism, requires challenging rectangular stems with blended fillets attached directly to walls, and the long thin plates may be tough to cool.

Electromagnetic simulations were performed in CST Microwave Studio. We optimized both the radius of the cavity and the electrode support length to match the resonant frequency and minimize RF losses. Shorter supports have a smaller surface therefore the $\mathrm{H}$-field density on it is higher, resulting in higher losses. When support length approaches cavity length, the $\mathrm{H}$-field density in the area between them increases resulting in higher losses. While there is an optimum value for the support length, it was decreased in order to reduce the cavity radius by $28 \%$ (see Fig. 6). Even in this case, we found that while the RF losses are low $(14.8 \mathrm{~kW})$, the tank diameter is unacceptably 

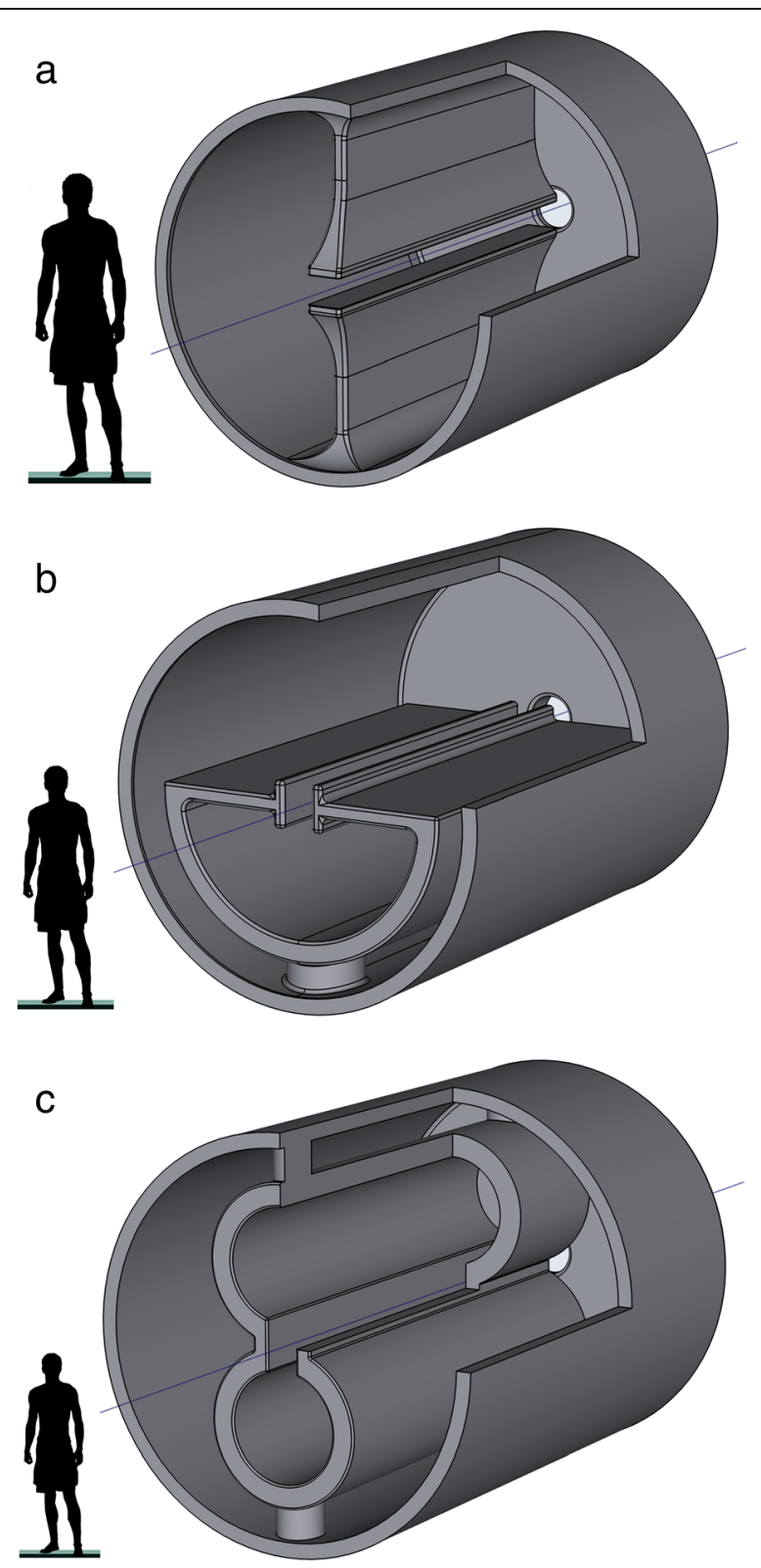

Fig. 5 Typical geometries of $\mathrm{H}-(\mathbf{a}), \mathrm{CH}-(\mathbf{b})$ and $8 \mathrm{H}-(\mathbf{c})$ resonators. Human silhouette is placed for scale

large $(2.7 \mathrm{~m})$ for fabrication and handling, making this cavity a poor candidate for the RF fragment separator.

Next, we considered a CH-resonator with a simple shape (Fig. 5b) to estimate dimensions, losses, and its feasibility for this application. Unfortunately, even the preliminary simulations revealed that the parameters of $\mathrm{CH}$-resonator are unfavorable: extremely large diameter $(3.7 \mathrm{~m})$, high losses $(47 \mathrm{~kW})$ and fabrication complexity, compared to the IH-resonator, make this option not worth further investigation. Finally, a double $\mathrm{CH}-$ or $8 \mathrm{H}-$ resonator option was considered (Fig. $5 \mathrm{c}$ ), however the parameters were also found to be completely unacceptable ( $5.4 \mathrm{~m}$ diameter with $89 \mathrm{~kW}$ losses).

Another efficient type of resonator with transverse E-field orientation is the so-called split-coaxial resonator (SCR), shown in Fig. 7, where two electrodes are attached from 

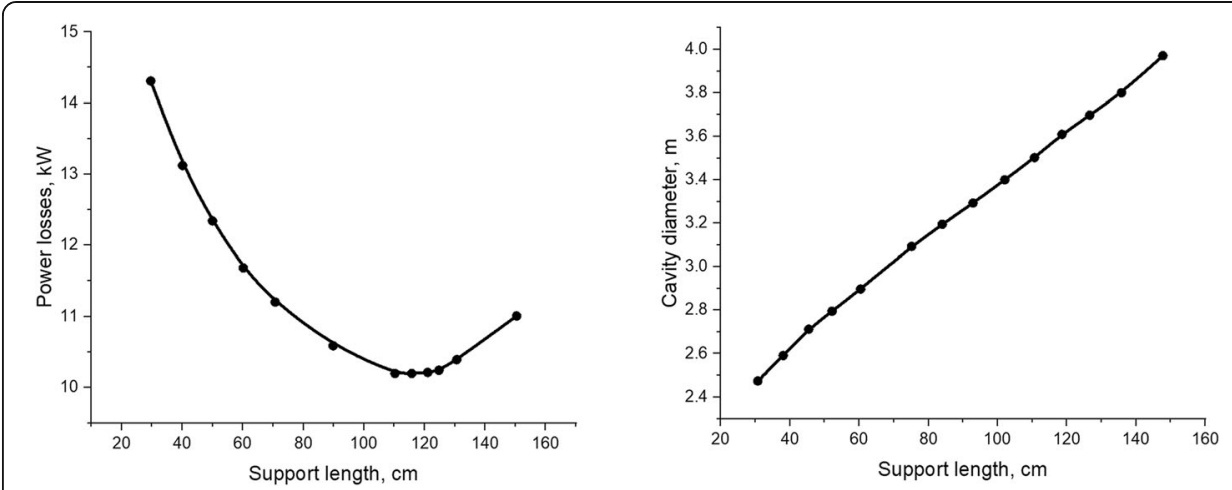

Fig. 6 RF losses (left) and optimal cavity radius (right) as functions of the electrodes support width (s) of $\mathrm{IH}$ resonator, simulated in CST Microwave Studio

different sides of a cylindrical cavity, and form a gap with an RF field in between them. This type of cavity is currently used in NSCL as a fragment separator [22]. The advantages of such resonators include high efficiency and the ability to minimize the tank dimensions by optimizing the electrode dimensions. A significant downside of this option is the sensitivity of the electrodes to vibrations, and of the large vacuum chamber for buckling, which is true for all large chambers. This cavity requires additional gussets to support plates, and the end flange parallelism is critical.

The first step of the cavity optimization was to identify RF parameter dependence of the SCR on the different dimensions. In particular, the cavity chamber radius $\left(R_{\text {cav }}\right)$ must be kept as low as possible, but results in higher frequency, which must be compensated by other dimensions. Wider $\left(t_{e l}\right)$ and thicker $\left(h_{e l 1}\right)$ electrodes improve the Qfactor due to lower surface $\mathrm{H}$-field density. However, the electric field strength on the beam axis drops significantly for wider electrodes, which dominates over the increase in Q-factor. The minimal width is defined by the field uniformity on the beam axis. After the elimination of the electrode width dimension $t_{e l}$ from the parameter optimization array, only 3 key dimensions are left: $R_{c a v}, h_{e l 1}$ and $k=h_{e l 1} / h_{e l 2}$. For smaller cavity chamber dimensions, electrodes should be as thin as possible. For lower losses, electrodes should be thick and the inclination should be increased. The optimal value can be found as a compromise between losses and dimensions as shown in Fig. 8 . Although the final diameter of the tank $(1.7 \mathrm{~m})$ is much smaller than the diameter of $\mathrm{IH}$-resonator, it is still too large for practical realization [23].

Finally, we considered quarter-wave resonators (QWR), shown in Fig. 9, which are used for low-beta SRF accelerators due to their simplicity and the fact that the resonant

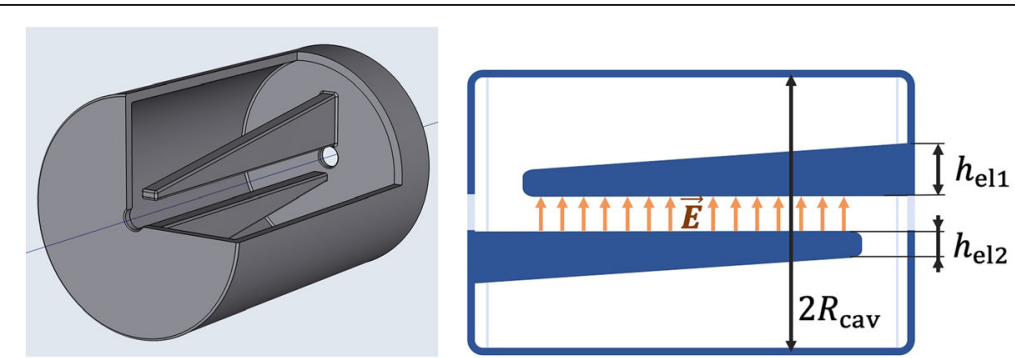

Fig. $73 \mathrm{D}$ (left) and schematic (right) models of split-coaxial resonator 


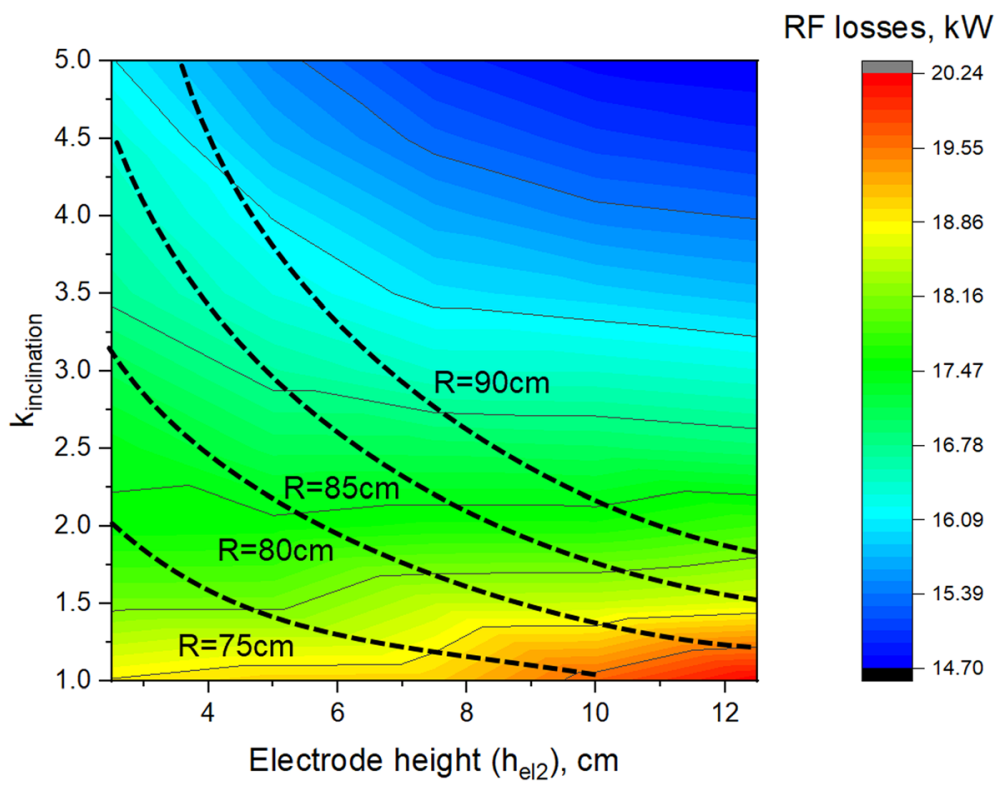

Fig. 8 RF losses in SCR as a function of electrode parameters for $20.125 \mathrm{MHz}$ frequency

frequency scales with the length of the inner conductor. Also, due to the fact that TEM-class waveguides do not have a cut-off frequency for the fundamental mode, the QWR diameter can be chosen to be very compact, limited only by the power handling capability.

From the mechanical point of view, QWRs can use out of vacuum kinematics on the top plate of the coaxial cavity for alignment and have easier access for plate alignment verification. The centrally located stem is good for weight distribution. On the other hand, the upper plate assembly can be challenging in-situ, the tapered 'tee' weldment is difficult to keep perpendicular and there is less room for tuners and couplers.

We also considered a double QWR (DQWR) option for the RF fragment separator. DQWR resembles a half-wave resonator (HWR), but unlike HWR, the phase of DQWR is push-pull rather than push-push. In general, in comparison to the QWR, DQWR option has a better field symmetry and lower RF losses, since for $300 \mathrm{kV}$ RF amplitude the individual electrodes operate as $0 / 300 \mathrm{kV}$ in the QWR case and $-150 /+150 \mathrm{kV}$ in the DQWR case. Intrinsically the DQWR would then operate at lower RF power by about

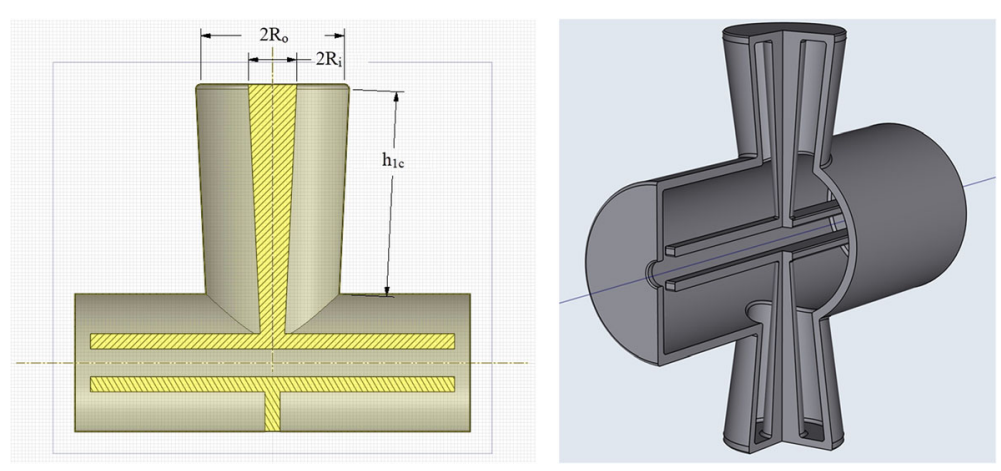

Fig. 9 Schematic model of single QWR (left) and preliminary 3D model of double QWR (right) 
a factor of 2 if no other changes were required. However, DQWR has twice larger vertical dimensions and requires shape optimization in order to fit into the FRIB beamline. For example, by reducing the length of the bottom coaxial by $27 \%$, the RF losses are increased by $38 \%$. Such a cavity can use vacuum kinematics on both plates for alignment. The centrally located stem is good for weight distribution, and this stem can also be used to feed internal cooling.

The most important QWR parameters to optimize are the radii of the inner and outer conductors $\left(R_{o}\right.$ and $\left.R_{i}\right)$. Their ratio defines the line impedance $Z=\frac{V^{2}}{P}=\sqrt{\frac{\mu}{\varepsilon}} \frac{\ln \left(R_{o} / R_{i}\right.}{2 \pi}$ should be kept higher to decrease losses. The frequency is defined by the length of the coaxial line $\left(h_{1 c}\right)$. By increasing the impedance, it is possible to decrease both losses and transverse size, however, the coaxial resonator size increases exponentially. An impedance of $Z=100 \Omega$ was selected to keep the coaxial diameter below $0.5 \mathrm{~m}$. RF losses can be further reduced by tapering the inner conductor [24].

The optimal parameters for all cavity types achieved during the preliminary electromagnetic design are compared in Table 1 . The weight, material and fabrication costs of the tanks are obviously proportional to their diameters. In addition, buckling and cooling problems also scale with the diameter. Given these considerations, we have excluded H-resonators from consideration, as unfeasible for fabrication. SCR appeared to be an attractive solution due to low-losses, but large diameter of the tank and problems with the electrodes bending have outweighted the benefits. Finally, choosing between QWR and DQWR, and taking into account FRIB experience in multiphysics and mechanical design of CW QWRs [25, 26], we decided to proceed with the detailed design and optimization of DQWR cavity, due to the lower losses and loss density, as well as EM field symmetry concerns.

\section{Design of DQWR cavity}

The model of the DQWR cavity is shown in Fig. 10. The design optimization of the interior shapes was done in consecutive steps. First, by adjusting the length of each vertical quarter-wave coaxial resonator we tuned the frequency to the specified 20.125 MHz. Second, we verified that the peak electric field strength is within the RF breakdown limit [27], i.e. $<1 \mathrm{Kp}=6.64 \mathrm{MV} / \mathrm{m}$ at $20.125 \mathrm{MHz}$. The E-field hotspot is located on the tips of the electrodes. It was reduced by slightly shortening the electrodes, thus increasing the gap between the electrode tip and the front wall of the rectangular cavity, as shown in Fig. 11, left. The electrodes have a small flat region due to the fabrication feasibility, since they are not solid but have an internal structure: please, refer to

Table 1 Comparison of different cavities parameters

\begin{tabular}{llll}
\hline Type & Tank diameter, $\mathbf{c m}$ & Q & RF losses, kW \\
\hline SCR & 170 & 21,500 & 21.3 \\
QWR & $60 / 80^{\mathrm{a}}$ & 17,500 & 38.6 \\
DQWR & $60 / 80$ & 14,000 & 34.0 \\
$\mathrm{IH}-$ & 270 & 26,000 & 14.3 \\
$\mathrm{CH}-$ & 370 & 17,500 & 47.0 \\
$8 \mathrm{H}-$ & 540 & 18,900 & 89.0 \\
\hline Tank / cavity & & &
\end{tabular}


the Engineering Design section for more details. Finally, we reduced the RF losses by optimizing the tapering and outer diameter of the coaxial resonator.

The final geometry has relatively narrow electrodes, which may affect the field uniformity. We estimated transverse field uniformity of $0.14 \%$ within the $\pm 1 \mathrm{~cm}$ range (four time larger than the realistic FWHM beam size of $0.5 \mathrm{~cm}$ [20]), as shown in Fig. 11, right, which is acceptable for fragment separation. Also, all the fragments going through the aperture have very similar transversal emittance: they are produced by the same fragmentation reaction and are selected by the same fragment separator with the same geometrical acceptances.

We performed CST Particle Studio beam dynamics simulations of ${ }^{100} \mathrm{Sn}^{50+}$ ions with $100 \mathrm{MeV} / \mathrm{u}$ energy to verify the required deflection. The simulation results, shown in Fig. 12, demonstrate that with $300 \mathrm{kV}$ voltage between electrodes, the beam deflection is $13.6 \mathrm{~mm}$, which is larger than the required deflection of $13 \mathrm{~mm}$. The corresponding simulated transverse angle is $8.8 \mathrm{mrad}$, larger than the required angle of $8.6 \mathrm{mrad}$. These results verify the feasibility of the RF design, assuming a realistic beam spot size of $5 \mathrm{~mm}$ (FWHM).

The results of the sensitivity analysis are presented in Table 2. For the anticipated average tolerance for each dimension of $1 \mathrm{~mm}$, the expected frequency deviation is estimated to be $\pm 40 \mathrm{kHz}$. The Lorentz detuning [28] is estimated to be withtin $700 \mathrm{~Hz}$, according to CST simulations. We designed a tuning mechanism for the DQWR cavity. Coarse tuning of the cavity will be performed by a sliding panel tuner, shown in Fig. 13,

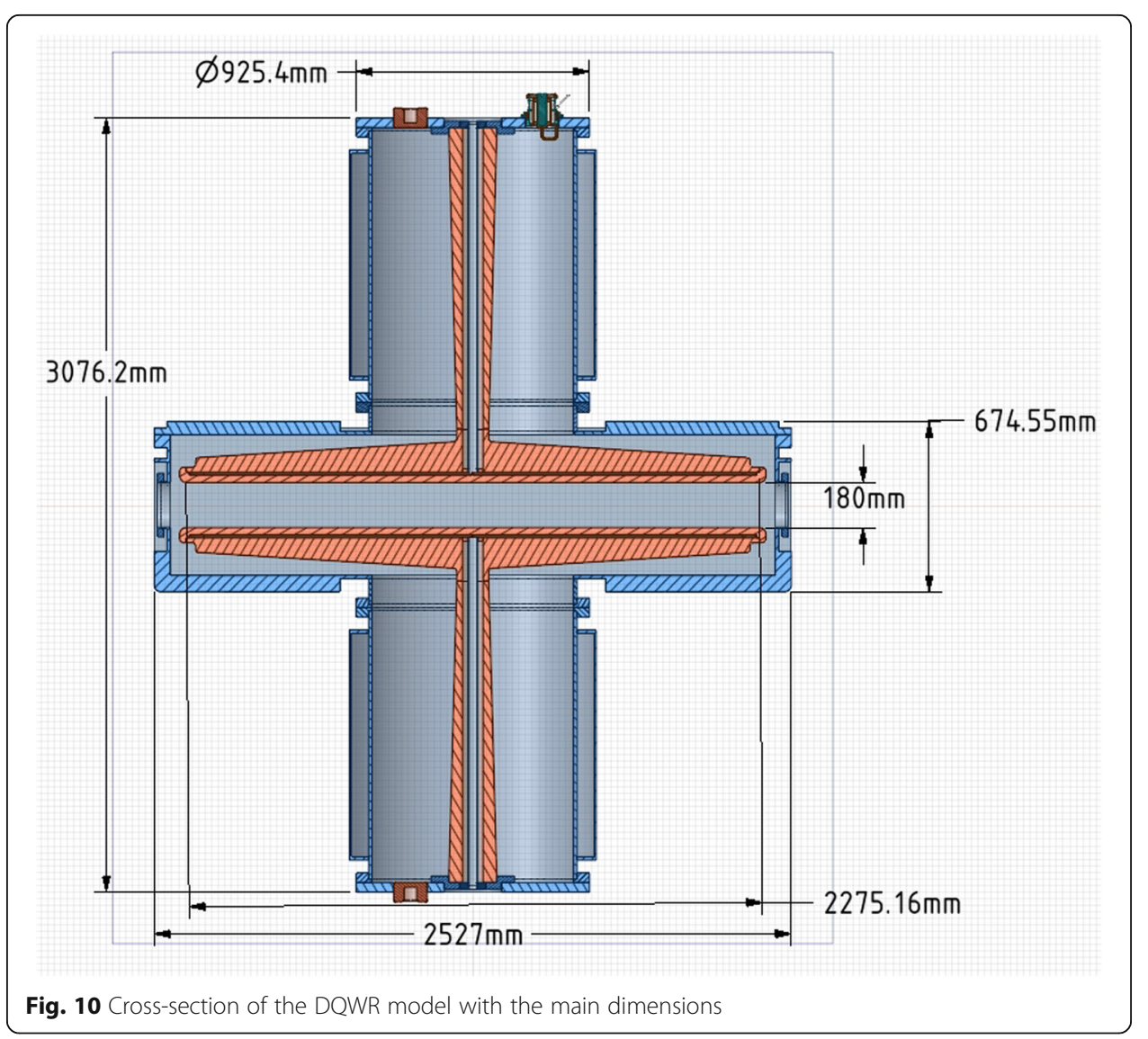



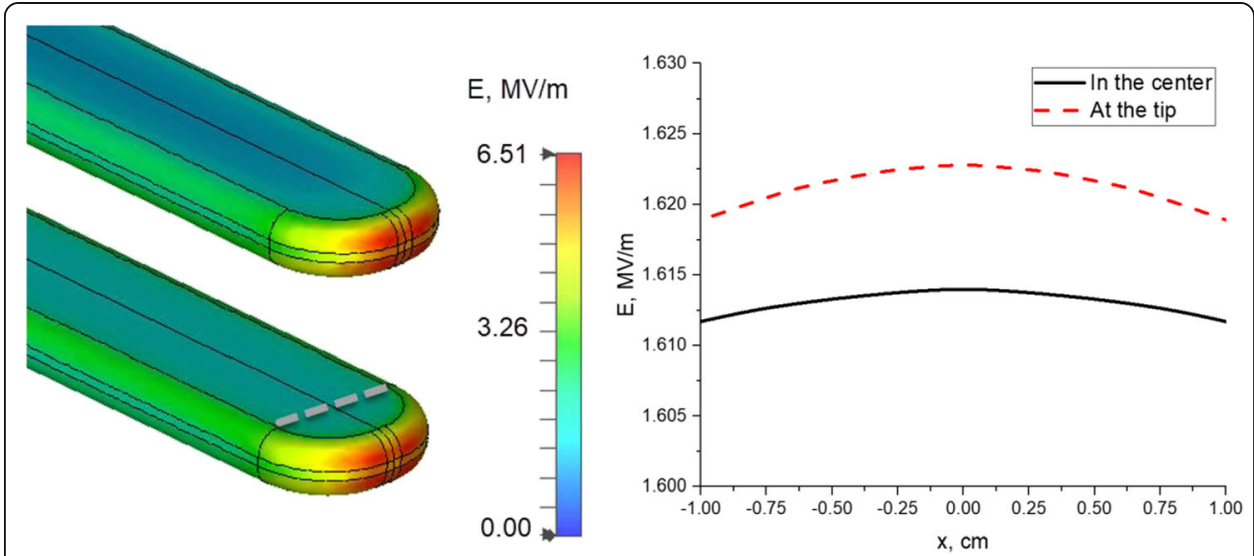

Fig. 11 Electric field distribution at the electrode tip, simulated in CST Microwave Studio (left), $6.51 \mathrm{MV} / \mathrm{m}$ corresponds to $0.98 \mathrm{Kp}$, and along the coordinate orthogonal to the deflection plane (right) at the center of the electrode (black) and near the electrode tip (red) to show field uniformity. The position of the latter path is defined, according to dashed line in the right figure, simulated in CST Particle Studio

right, which allows tuning sensitivity of $\sim 30 \mathrm{kHz} / \mathrm{cm}$. For dynamic tuning to cover thermal expansion effects, we considered using a slug tuner, similar to those for the ATLAS RFQ [29], on the opposing arm, as shown in Fig. 13, left. The resulting sensitivity of $\sim 1.6 \mathrm{kHz} / \mathrm{cm}$ per tuner for the full movement range, providing a $\pm 6 \mathrm{kHz}$ range.

We also completed the design of the RF coupler. We chose a modified version of the water-cooled magnetic-loop coupler as used in the ATLAS RFQ [29]. This coupler can operate at $60 \mathrm{~kW}$ power, so it is a good match for the application in the DQWR. The electromagnetic simulations demonstrated that in order to critically couple the structure, the coupling loop length should be increased by $8 \mathrm{~cm}$ as shown in Fig. 14. Typically, the design coupling strength is chosen slightly above $1.0(\sim 1.1-1.2)$, to ensure that the cavity is not undercoupled at any operation conditions [30].

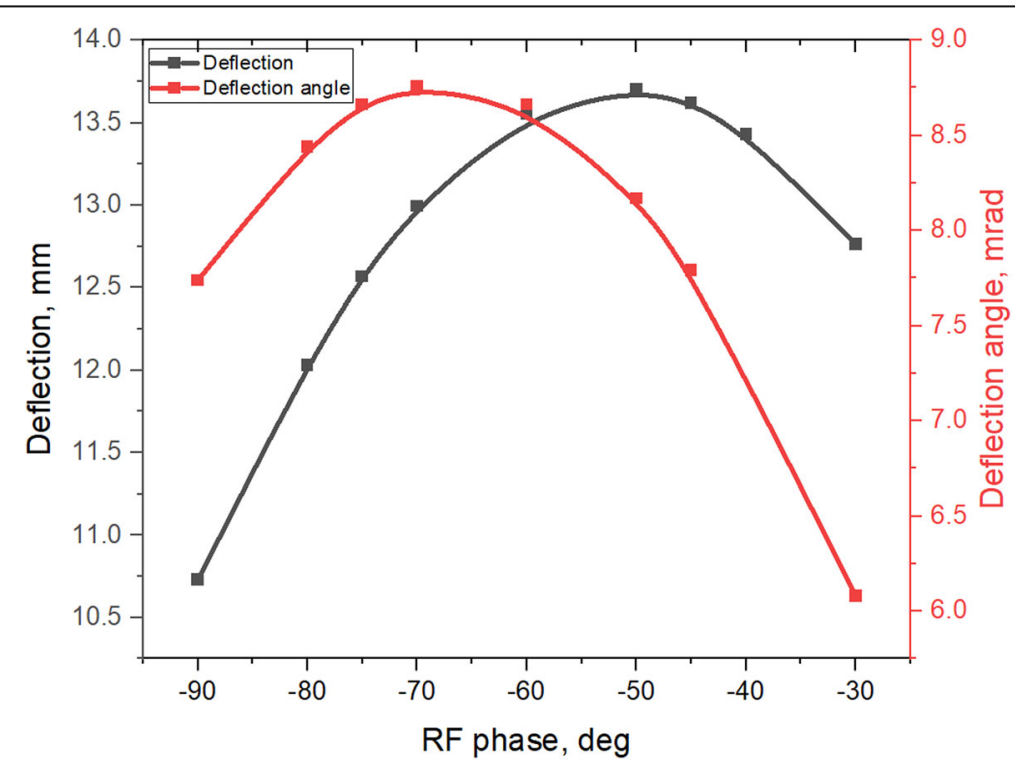

Fig. 12 Deflection and angle after $3 \mathrm{~m}$ from the entrance to the cavity as a function of particle phase, relative to RF as simulated in CST Particle Studio 
Table 2 Frequency sensitivities to the geometrical dimensions

\begin{tabular}{ll}
\hline Dimension & Sensitivity, $\mathbf{k H z} / \mathbf{m m}$ \\
\hline Tank diameter & 17.1 \\
Stem top diameter & -6.4 \\
Stem bottom diameter & 15.3 \\
Electrode length & -8.5 \\
Tank length & -7.7 \\
Electrode width & -24.0 \\
Gap width & 17.1 \\
\hline
\end{tabular}

\section{Engineering design}

Advanced conceptual engineering design was performed on the DQWR to ensure manufacturability, baseline structural robustness and to verify the required RF sensitivities are within cavity manufacturing tolerances. The DQWR efforts can be broken into 3 separate sub-systems: the electrodes/stem, the main chamber, and coupling/tuning sub-assemblies.

The baseline electrode/stem assembly was addressed by first ensuring adequate stiffness along the electrode longitudinal extent in the fully annealed state. As can be seen in Fig. 15, the electrodes demonstrated nearly $0.5 \mathrm{~mm}$ of deflection at the furthest extents purely under a weight load. This was mitigated through the addition of a gusset reducing this deflection to $<120 \mu \mathrm{m}$. The next feature set addressed in the design was the water cooling required to cool the electrodes and stems. By utilizing an inner/outer concentric tube concept to introduce water down the stem and into the electrodes, a significant amount of cooling capacity was obtained, while not sacrificing the structural integrity of the sub-assembly. The introduction of cooling into the electrode will necessitate a water to vacuum braze joint, which is designed with both lap and shear joints for strength and hermetic redundancy. However, there are examples [31, 32] of the operational structures with such brazing, and we believe that it won't cause any problems in this particular design.

The cavity will be constructed from 304 stainless steel (SS) and copper-plated afterwards. The cavity will be split into 4 major weldments and constructed as a central rectangular chamber with cylinders on the top and bottom. Vacuum seals are accomplished with Viton O-rings and RF contact is accomplished with Balseal canted coil RF springs. Significant efforts were placed on designing the central box while keeping the deflections from vacuum forces and gravity $<0.5 \mathrm{~mm}$ and the stresses below the yield for 304SS. As shown in Fig. 16, the deformations were $<0.4 \mathrm{~mm}$ and stresses a factor of
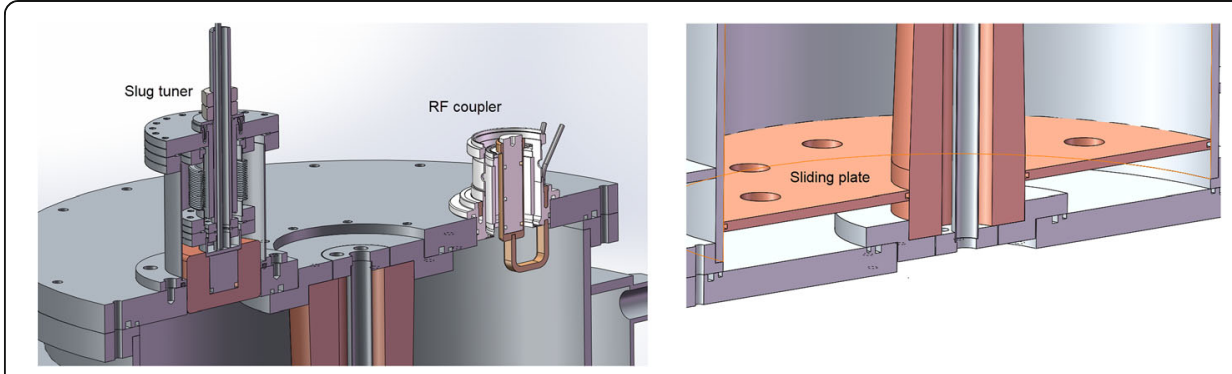

Fig. 13 Slug tuner (left) and sliding plate tuner (right) 

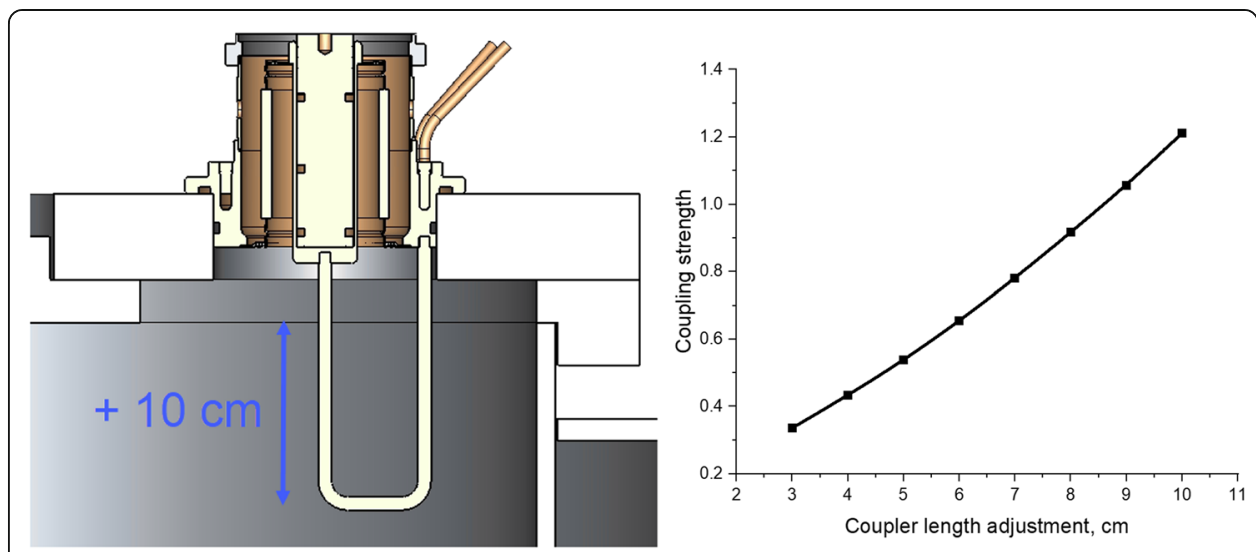

Fig. 14 Magnetic RF coupler attached to the DQWR (left) and simulated coupling strength as a function of the loop length increase (right)

3 below the yield. The upper and lower cylindrical chambers required no rib features for structural stability; however, some features have been included in the outer wall of the vacuum chamber to assist in flow dynamics around the cooling jackets.

Next, preliminary modal analysis was performed to identify regions where simple features could stiffen the chamber. As a starting point, the tank was held at the four support points. We assumed a completely rigid support system and only examined the resonant modes of the device itself. These iterations ultimately resulted in a chamber with no vibrational modes below $40 \mathrm{~Hz}$, a generally acceptable result as high-frequency modes dissipate sooner in building foundations. Therefore the amplitudes of these excitations are smaller at higher frequencies and thus less likely to affect the structure. However, further efforts prior to fabrication are required to take into consideration a realistic kinematic stand, in order to minimize the high-amplitude coupled modes
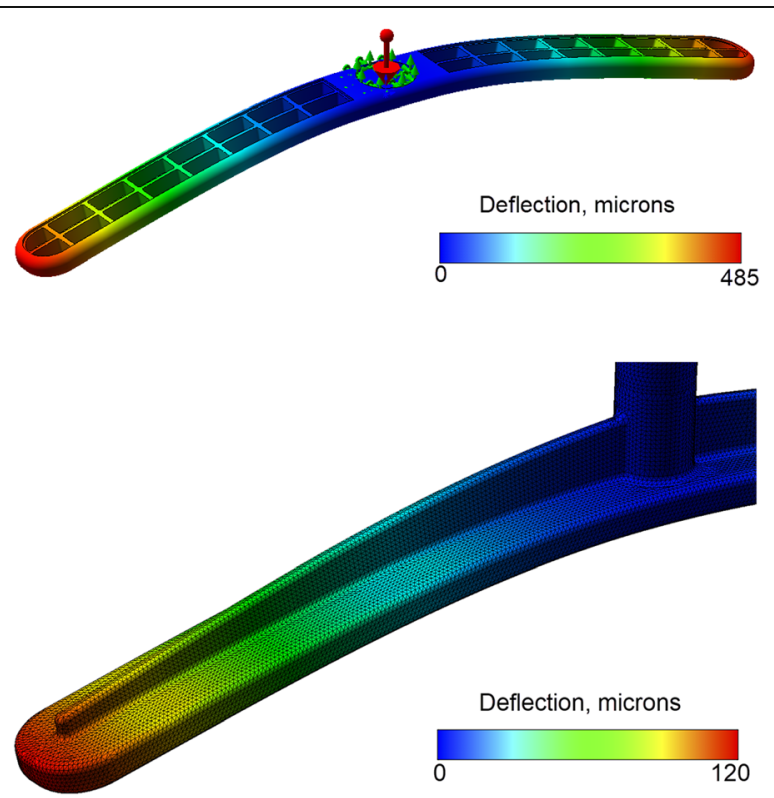

Fig. 15 Electrode assembly evolution; starting point with $\sim .5 \mathrm{~mm}$ deflection (top) and final support gusset to reduce deflection to $\sim 120 \mu \mathrm{m}$ (bottom) as simulated in SolidWorks 
between the cavity and the stands and consider any frequency mapping of the proposed installation site to ensure modal stability. We have also performed the preliminary thermal analysis of the cavity with different cooling options in CST Multiphysics Studio. Figure 17 presents the model, used for simulations with the assumed thermal boundary conditions. The heat transfer coefficient was calculated in ANSYS CFX, considering 50 $1 / \mathrm{m}$ water flow rate as shown in Fig. 18. The simulation results demonstrate that the structure can be effectively cooled by using three cooling circuits: inner electrode cooling channels, water jacket around the coaxial resonator, and cooling tubes on the vacuum chamber.

\section{Results and discussion}

We have designed an efficient fragment separator for high energy secondary radioactive beams, including the electromagnetic and engineering design of the cavity. We optimized the cavity to minimize the power requirements, its cost and dimensions. All aspects of the cavity performance were verified through multiphysics computer simulations. The optimized design offers several attractive features:

- The use of two coaxial resonators allows high voltage operation with reasonable RF power while reducing the vacuum chamber dimensions to an acceptable level for fabrication feasibility;

- The higher impedance of QWRs and the smaller dimension of the coaxial line reduce the required power and made the design more compact;

- Optimized electrode shape allows high voltage operations with peak fields under 1 Kp, provides good structural stability;

- Power coupler optimization allows operation with only a single coupler;

- Tuning mechanisms allow precise tuning of the cavity over a wide frequency range;

- The cooling system allows thermal and frequency stability of the cavity;
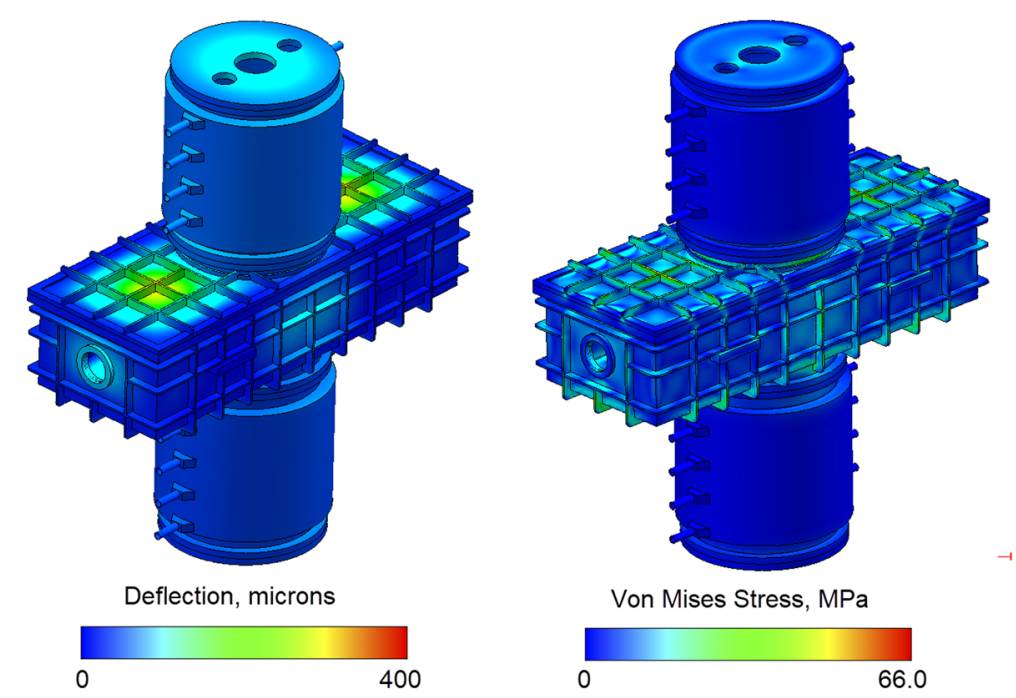

Fig. 16 Deformation (left) and VonMises stress (right) results of simulations on the cavity engineering model demonstrating $<0.5 \mathrm{~mm}$ wall deformation and no major yield issues as simulated in SolidWorks 


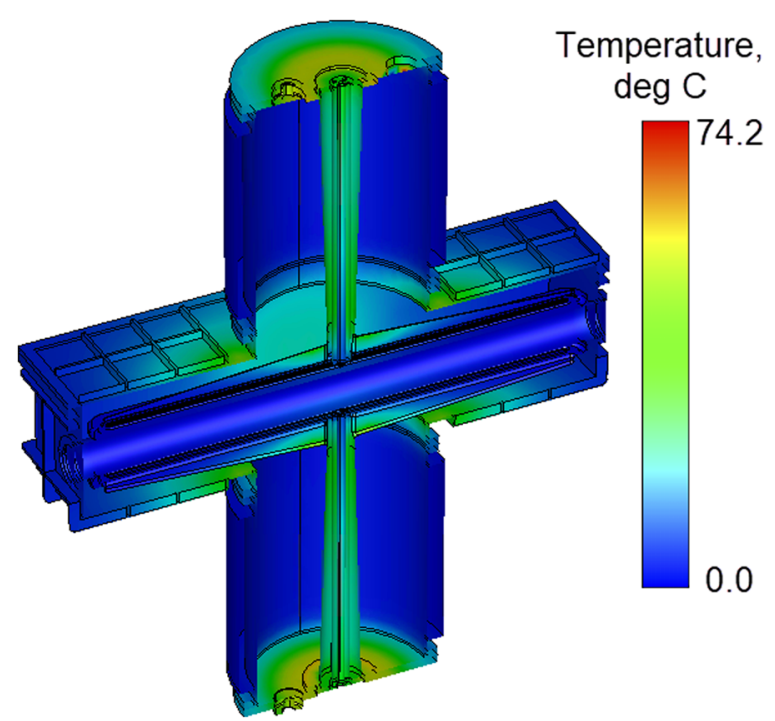

Fig. 17 Temperature map of the DQWR separator cavity under full RF power load as simulated in CST Multiphysics Studio

In addition to the direct application to FRIB, the technology developed in this project can be applied to other heavy-ion machines. RF fragment separators are also of interest for mass-spectrometry as they can provide very precise isotope separation for security, defense, and environmental applications.

\section{Conclusion}

In this work we have demonstrated the feasibility of RF cavity design for FRIB radioactive isotopes separation and estimated the required and actual parameters of the RF cavity. This work will serve as a ground for the future prototype fabrication and will initiate the design of other related subcomponents such as RF chopper.
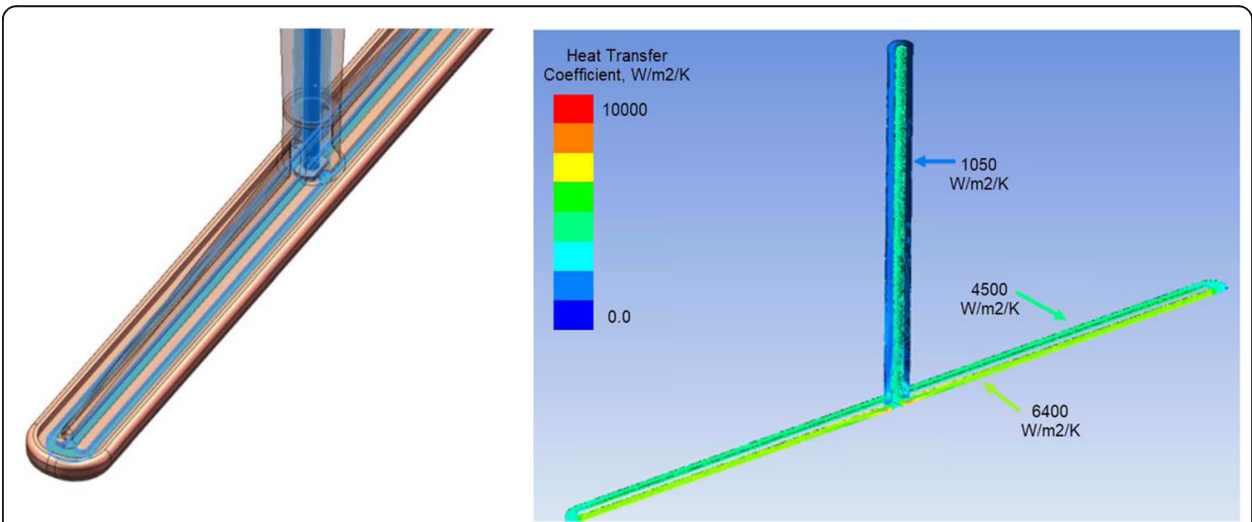

Fig. 18 Internal geometry of electrode cooling channels (left) and simulated heat transfer coefficient map inside the cooling channels for $50 \mathrm{l} / \mathrm{s}$ water flow (right) as simulated in ANSYS CFX 


\section{Abbreviations}

ATLAS: Argonne Tandem Linear Accelerator System; CFD: Computational fluid dynamics; CST: Computer Simulation Technologies; CW: Continuous wave; DQWR: Double Quarter-Wave Resonator; FAIR: The Facility for Antiproton and Ion Research; FRIB: The Facility for Rare Isotopes Beams; FWHM: Full width half maximum; HWR: Half-wave resonator; MSU: Michigan State University; NSCL: The National Superconducting Cyclotron Laboratory; QWR: Quarter-wave resonator; RF: Radio Frequency; RFFS: RF Fragment Separator; RFQ: Radio Freuquency Quadrupole; RIB: Rare isotope beam; RIBF: Radioactive Ion Beam Factory; SCR: Split coaxial resonator; SRF: Superconducting RF; SS: Stainless steel; TE: Transverse Electric field mode; TEM: Transverse electromagnetic mode

\section{Authors' contributions}

The resonator design and optimization were done by SK, AP, AS and KT. The resonator parameters in view of FRIB were defined by RZ and PO. Beam dynamics simulations were performed by DB and OT. Engineering design were performed by RA and NB. The project was led by SK on behalf of RB and PO on behalf of FRIB. All authors commented on the manuscript. All authors have read and approved the final manuscript.

\section{Funding}

This work was supported by the US Department of Energy, Office of Nuclear Physics, under SBIR grant DE-SC0019723.

\section{Availability of data and materials}

The datasets used and/or analyzed during the current study are available from the corresponding author on reasonable request.

\section{Competing interests}

The authors declare that they have no competing interests.

\section{Author details}

${ }^{1}$ RadiaBeam Technologies LLC, 1717 Stewart St, Santa Monica, CA 90404, USA. ${ }^{2}$ Facility for Rare Isotope Beams, Michigan State University, 640 S Shaw Ln, East Lansing, MI 48824, USA. ${ }^{3}$ National Superconducting Cyclotron Laboratory, Michigan State University, 640 S Shaw Ln, East Lansing, MI 48824, USA. ${ }^{4}$ Department of Physics and Astronomy, Michigan State University, 567 Wilson Road, East Lansing, MI 48824, USA. ${ }^{5}$ Joint Institute for Nuclear Astrophysics, Center for the Evolution of the Elements, Michigan State University, 640 S Shaw Ln, East Lansing, MI 48824, USA.

Received: 17 August 2020 Accepted: 10 November 2020

Published online: 01 December 2020

\section{References}

1. Morrissey DJ, Sherrill BM. Radioactive nuclear beam facilities based on projectile fragmentation. Philos Trans R Soc Lond A. 1998:356:1744

2. Nolen J. Viewpoint: Pushing Back the Frontier of Stability. Physics. 2013;6:59.

3. Fukunishi N, et al. "Present Performance and Commissioning Details of RIBF Accelerator Complex", Cyclotrons and Their Applications 2007, Eighteenth International Conference.

4. Kester O, et al. "Status of the FAIR Accelerator Facility", Proceedings of IPAC2014, Dresden, Germany.

5. Wei J, et al. Advances of the FRIB project. Int J Modern Physics E. 2019;28(3):1930003.

6. FRIB Users Organization for the NSAC Long Range Plan Implementation Subcommittee. "FRIB: Opening New Frontiers in Nuclear Science. Moving Forward with the Long Range Plan", August 2012, available at: https://frib.msu.edu/_files/pdfs/ frib_opening_new_frontiers_in_nuclear_science.pdf.

7. National Research Council. Nuclear Physics: exploring the heart of matter. Washington, DC: The National Academies Press; 2013.

8. Aprahamian A, et al. "Reaching for the horizon: The 2015 long range plan for nuclear science", Reaching for the Horizon: The 2015 Long range plan for nuclear science. United States: N. p., 2015.

9. Mantica P. Facility for Rare Isotope Beams and National Superconducting Cyclotron Laboratory Overview; 2015.

10. Bandura L. "Next-Generation Fragment Separators for Exotic Beams", Ph.D. Thesis, Northern Illinois University; 2009.

11. Morrissey DJ. Status of the FRIB project with a new fragment separator. J Phys Conf Ser. 2011;267:012001.

12. Morrissey DJ, et al. Commissioning the A1900 projectile fragment separator. Nucl. Instr. and Meth. B. 2003;204:90

13. Yamada K, et al. RF deflector system for proton-rich RI beams in RIKEN. Nucl Phys A. 2004;746:156.

14. Blackmore EW, et al. An RF separator for cloud muons at TRIUMF. Nucl Instr And Meth, Phys Res A. 1985;234:235

15. Baumann T. "The Radio Frequency Fragment Separator: A Time-of-Flight Filter for Fast Fragmentation Beams", presented at Cyclotrons '13 Vancouver 2013-09-18FR1PB03.

16. Morris D, et al. "RF System for FRIB Accelerator", Proc. of IPAC2018, Vancouver, BC, Canada; 2018. p. WEXGBF3.

17. Tarasov OB, Bazin D. LISE++: Radioactive beam production with in-flight separators. Nucl Instr And Meth Phys Res B. 2008:266:4657.

18. Bazin D, et al. The program LISE: a simulation of fragment separators. Nucl Instrum Meth Phys Res A. 2002;482:307.

19. Tarasov OB, Bazin D. LISE++: exotic beam production with fragment separators and their design. Nucl Instr Meth Phys Res B. 2016:376:185.

20. Zegers R. "FRIB High Rigidity Spectrometer", Conceptual Design Report, FRIB-M41800-AD-000531-R002; 2019

21. Wangler T. Principles of RF linear accelerators. New York: Wiley; 1998. p. 382

22. Bazin D, et al. Radio frequency fragment separator at NSCL. Nucl Inst Methods Phys Res A. 2009;606:314-9.

23. The American Society of Mechanical Engineers. 2017 ASME Boiler \& Pressure Vessel Code. VIII: Rules for Construction of Pressure Vessels Division 1. New York: ASME; 2017. 
24. Mustapha B, et al. "Electro-Magnetic Optimization of a Half-Wave Resonator", Proceedings of SRF2011, Chicago, IL USA; 2011. p. MOPO044.

25. Pozdeyev E, et al. FRIB Front End Design Status, Proc. 26th Linear Accelerator Conf. (LINAC'12), Tel Aviv, Israel; 2012. p. THPB097.

26. Plastun AS, et al. Longitudinal Beam Dynamics in FRIB and ReA Linacs, Proc. 13th international computational accelerator physics conference (ICAP'18), Key West, Florida, USA; 2018. p. 330-4

27. Kilpatrick WD. Criterion for Vacuum Sparking Designed to Include Both if and dc. Rev Sci Instrum. 1957;28(10):824-6.

28. Verdú-Andrés S, et al. "Lorentz Detuning for a Double-Quarter Wave Cavity", Proceedings of SRF2015, Whistler, BC, Canada, pp. THPB051.

29. Kutsaev S, et al. Design and multiphysics analysis of a $176 \mathrm{MHz}$ continuous-wave radio-frequency quadrupole. Phys Rev ST Accel Beams. 2014;17:072001.

30. Kutsaev S, et al. Four-harmonic buncher for radioactive and stable beams switching at the ATLAS facility. Nucl Inst Methods Phys Res A. 2018:905:149-59.

31. Rathke JW, et al. Preliminary Engineering Design of A $57.5 \mathrm{MHz}$ CW RFQ for The RIA Driver LINAC, Proc. 21st Linear Accelerator Conf. (LINAC'02), Gyeongju, Korea; 2002. p. TU466.

32. Zeng J, et al. Cooling Design for the FRIB RFQ Cavity at Michigan State University, Proc. 5th Int. Particle Accelerator Conf. (IPAC'14), Dresden, Germany; 2014. p. THPME024.

\section{Publisher's Note}

Springer Nature remains neutral with regard to jurisdictional claims in published maps and institutional affiliations.

Submit your manuscript to a SpringerOpen ${ }^{\circ}$ journal and benefit from:

- Convenient online submission

- Rigorous peer review

- Open access: articles freely available online

- High visibility within the field

- Retaining the copyright to your article

Submit your next manuscript at $>$ springeropen.com 